\title{
Die Anwendung der rotierenden Kathode zur Bestimmung des Kadmiums aus seinen Chloridlösungen.
}

\section{Von}

\author{
Charles P. Flora. ${ }^{1}$
}

In der vorstehenden Mitteilung ${ }^{2}$ ist die Anwendung der rotierenden Kathode zur Bestimmung von Kadmium aus den Lösungen seines Sulfats beschrieben worden; eine ähnliche Untersuchung über das Verhalten des Kadmiums in seinen Chloridlösungen soll hier mitgeteilt werden. Einige Unterschiede sind zu erwarten, da mit Sicherheit festgestellt ist, dals Kadmiumchlorid bei der Elektrolyse nicht nur positive Kadmium- und negative Chlorionen bildet, sondern auch komplexe negative Kadmiumchlorionen: aufserdem zersetzt das Chlor nach seiner Abscheidung nicht direkt das Wasser unter Bildung von Sauerstoff, sondern es tritt frei in der Lösung auf. Dals sich hierdurch sehr wichtige Unterschiede geltend machen, zeigten einige qualitative Versuche, und deswegen wurde die elektrolytische Bestimmung des Kadmiums aus seinen Chloridlösungen näher untersucht.

Es wurde eine Lösung von geeigneter Konzentration hergestellt und ihr Gehalt durch verschiedene gut übereinstimmende Bestimmungen nach der Karbonatmethode festgestellt; diese Methode war besonders geprüft und als zuverlässig befunden worden. $30 \mathrm{ccm}$ der Lösung enthielten $0.1589 \mathrm{~g}$ Kadmium, demnach enthielt $1 \mathrm{ccm}$ $0.0052966 \mathrm{~g}$, Metall.

1 Aus dem American Journal of Science (Silliman) ins Deutsche übertragen von J. KopPEx.

2. anorg. Chem. 47, 1. 


\section{Bestimmangen in schwefelsäurehaltigen Lösungen.}

Dies Verfahren wurde beim Chlorid ebenso ausgeführt wie beim Sulfat; die Resultate waren sehr zufriedenstellend. Es ist besonders darauf zu achten, dafs die Verdünnung richtig gewählt wird; denn aus verdünnteren Lösungen ist es fast unmöglich, die letzten Spuren des Metalles zu entfernen. Bei einer Verdünnung von $45 \mathrm{ccm}$ erhielt man die besten Resultate. Vor der Elektrolyse wurde die Lösung mit 10 Tropfen Schwefelsäure $(1: 4)$ versetzt. Unter diesen Bedingungen ergaben sich die folgenden Zahlen.

\begin{tabular}{c|c|c|c|c|c|c|c}
\hline \hline Nr. & $\begin{array}{c}\text { Angew. } \\
\text { Cd in g }\end{array}$ & $\begin{array}{c}\text { Stromstärke } \\
\text { in Amp. }\end{array}$ & $\begin{array}{c}\text { N. D.100 } \\
\text { in Amp. }\end{array}$ & $\begin{array}{c}\text { E.M.K. } \\
\text { in Volt }\end{array}$ & $\begin{array}{c}\text { Zeit } \\
\text { in Min. }\end{array}$ & $\begin{array}{c}\text { Gef. Cd } \\
\text { in } \mathbf{g}\end{array}$ & $\begin{array}{c}\text { Fehler } \\
\text { in } \mathbf{g}\end{array}$ \\
\hline 1 & 0.1059 & $1.0-1.5$ & $3.0-4.5$ & $6.5-7.8$ & 25 & 0.1054 & -0.0005 \\
2 & 0.1059 & $2.0-3.0$ & $6.0-9.0$ & 7.8 & 15 & 0.1058 & -0.0001 \\
\hline
\end{tabular}

II. Bestimmungen in acetathaltigen Lösungen.

Die Acetatmethode hat sich bei der Bestimmung von Kadmiumsulfat als eine der zufriedenstellendsten erwiesen, aber merkwürdigerweise ist sie gänzlich ungeeignet zur Elektrolyse des Metalles aus dem Chlorid. Das abgeschiedene Kadmium war immer schwammig, oft nicht fest haftend und liefs sich nicht quantitativ behandeln. Die „Schwammbildung“ war weniger ausgesprochen, wenn kein Kaliumsulfat vorhanden war, aber auch dann haftete das Metall nicht und liefs sich nicht zur Wägung bringen. Die versuchten Abänderungen der Methode sind zum Vergleich in der folgenden Tabelle zusammengestellt. Die Stromspannung betrug durchweg 7.8 Volt, die Verdünnung - aufser bei Nr. $4-45 \mathrm{ccm}$. Bei Versuch 4 wurde mit $65 \mathrm{ccm}$ gearbeitet, doch war kein Vorteil bemerklich.

\begin{tabular}{|c|c|c|c|c|c|c|c|}
\hline 妾 & 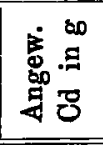 & 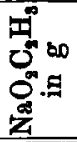 & 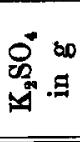 & 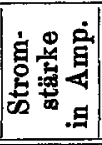 & 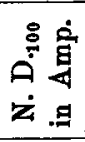 & 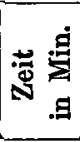 & Bemerkungen \\
\hline 1 & 0.1324 & 1.5 & 0.5 & 1.5 & 4.5 & 13 & sehr schwummig \\
\hline 2 & 0.1324 & 1.5 & - & 1.0 & 3.0 . & 8 & $0.1314 \mathrm{~g}$ gefunden \\
\hline $\mathbf{3}$ & 0.1059 & 1.0 & - & 0.75 & 2.25 & 20 & nicht haftend \\
\hline 4 & 0.1059 & 1.5 & 0.5 & 1.5 & 4.5 & - & schwammig, nicht haftend \\
\hline 5 & 0.1059 & 1.5 & - & 1.0 & 3.0 & - & 2 ccm Formalin zugesetzt; schw. \\
\hline 6 & 0.1059 & 1.5 & - & 0.75 & 2.25 & - & nicht haftend; kristallinisch \\
\hline 7 & 0.1059 & 0.5 & 0.5 & 1.0 & 3.0 & - & $"$ \\
\hline
\end{tabular}


III. Bestimmungen in cyanidhaltigen Lösungen.

Die Anwendung cyanidhaltiger Lösungen gab beim Chlorid ebenso zufriedenstellende Resultate wie beim Sulfat. Wie dort, so muls auch hier das Schäumen der Flüssigkeit vermieden werden. Die passendste Verdünnung schien $65 \mathrm{ccm}$ zu. sein. Die erforderliche Zeitdauer ist etwas länger als beim Sulfat. Folgende Werte wurden erhalten:

\begin{tabular}{c|c|c|c|c|c|c|c|c|c|}
\hline \hline Nr. & $\begin{array}{c}\text { Angew. } \\
\text { Cd in } \mathrm{g}\end{array}$ & $\begin{array}{c}\text { KCN } \\
\text { in } \mathrm{g}\end{array}$ & $\begin{array}{c}\text { NaOH } \\
\text { in } \mathrm{g}\end{array}$ & $\begin{array}{c}\text { Stromst. } \\
\text { in Amp. }\end{array}$ & $\begin{array}{c}\text { N. D.100 } \\
\text { in Amp. }\end{array}$ & $\begin{array}{c}\text { E.M.K. } \\
\text { in Volt }\end{array}$ & $\begin{array}{c}\text { Zeit } \\
\text { in Min. }\end{array}$ & $\begin{array}{c}\text { Gef. Cd } \\
\text { in g }\end{array}$ & $\begin{array}{c}\text { Fehler } \\
\text { in g }\end{array}$ \\
\hline \\
\hline
\end{tabular}

Bei Versuch Nr. 2 trat starkes Schäumen ein und eine Spur Kadmium blieb in Lösung, da die Fällung sehr verzögert war.

\section{Bestimmungen in Lösungen von Pyrophosphaten.}

Die verschiedenen Abarten der Pyrophosphatmethode gaben ganz zufriedenstellende Resultate, die in jeder Beziehung vergleichbar waren mit den Bestimmungen von Kadmiumsulfat in demselben Elektrolyten. Wie dort, so wurden auch hier durch Auflösen des Niederschlages in Ammoniumbydroxyd die besten Werte erhalten; an zweiter Stelle schien Schwefelsäure ein geeignetes Lösungsmittel żu sein.

Die Niederschläge aus Lösungen mit freier Phosphorsäure zeigten geringe Neigung, schwammig zu werden. Nach Zusatz von Chlorwasserstoffsäure wurden die Metallschichten gut, aber die Fällung erfolgte nur langsam. Das Gesamtrolumen betrug in jedem Falle $45 \mathrm{ccm}$. $9.5 \mathrm{~g}$ Natriumpyrophosphat wurden verwendet. Die Stromspannung betrug 78 Volts.

Die folgenden Resultate wurden erhalten:

\begin{tabular}{|c|c|c|c|c|c|c|c|}
\hline : & $\begin{array}{c}\text { Angew. } \\
\text { Cd } \\
\text { in } g\end{array}$ & Lösungsmittel & $\begin{array}{l}\text { Strom- } \\
\text { stärke } \\
\text { in Amp. }\end{array}$ & $\begin{array}{l}\text { N. D.100 } \\
\text { in Amp. }\end{array}$ & 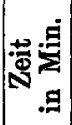 & 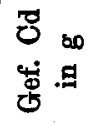 & 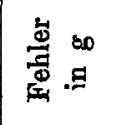 \\
\hline 1 & 0.1324 & $\mathrm{NH}_{4} \mathrm{OH}$, konz., $1 \mathrm{ccm}$ & 0.5 & 1.5 & 15 & 0.1327 & +0.0003 \\
\hline 2 & 0.1324 & $\mathrm{H}_{2} \mathrm{SO}_{4},(1.4), 12$ Tropfen & 0.75 & 2.25 & 35 & 0.1328 & +0.0004 \\
\hline $\mathbf{3}$ & 0.1324 & $\mathrm{H}_{8} \mathrm{PO}_{4}$ (sp. Gw. 1.7), 15Tpf. & $0.75-1.0$ & $2.25-3.0$ & 30 & 0.1331 & +0.0007 \\
\hline 4 & 0.1324 & $\mathrm{HCl}, 1: 4,15$ Tropfen. & $0.7-0.5$ & $2.1-1.5$ & 45 & 0.1319 & -0.0005 \\
\hline
\end{tabular}




\section{v. Bestimmungen in phosphathaltigen Lösungen.}

Beim Kadmiumchlorid mufs Dinatriumhydrophosphat mit noch mehr Sorgfalt benutzt werden als beim Sulfat, wenn nur. einigermafsen brauchbare Niederschläge erhalten werd̉en sollen; selbst bei grofser Vorsicht jedoch ist die Neigung zur Abscheidung schwammigen Metalles so hervortretend, dafs diese Methode nicht empfehlenswert erscheint, wenn andere anwendbar sind.

Im folgenden sind die benutzten Lösungen zusammengestellt; das Volumen betrug immer $45 \mathrm{ccm}$.

\begin{tabular}{|c|c|c|c|c|c|c|c|c|c|}
\hline Nr. & $\begin{array}{c}\text { Angew. } \\
\text { Cd } \\
\text { in } \mathrm{g}\end{array}$ & 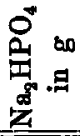 & $\begin{array}{c}\mathrm{H}_{8} \mathrm{PO}_{4} \\
(\mathrm{sp} \cdot \mathrm{G} .1 .7) \\
\text { in ccm }\end{array}$ & $\begin{array}{l}\text { Strom- } \\
\text { stärke } \\
\text { in Amp. }\end{array}$ & $\begin{array}{l}\text { N. D.100 } \\
\text { in Amp. }\end{array}$ & $\begin{array}{l}0 \\
0\end{array}$ & 莺苞 & $\begin{array}{c}\text { Gef. } \\
\text { Cd } \\
\text { in } \mathbf{g}\end{array}$ & $\begin{array}{c}\text { Fehler } \\
\text { in } g\end{array}$ \\
\hline 1 & 0.1059 & 0.25 & $5 \mathrm{ccm}$ & $2.0-3.0$ & $6.0-9.0$ & 7.8 & 15 & 0.1082 & +0.0023 \\
\hline 2 & 0.1324 & 0.25 & $2.5 \mathrm{ccm}$ & $2.0-3.0$ & $6.0-9.0$ & 7.8 & 13 & 0.1344 & +0.0020 \\
\hline $\mathbf{3}$ & 0.1324 & 0.20 & 10 Tropf. & 1.0 & $\mathbf{3 . 0}$ & 7.8 & 15 & 0.1330 & +0.0006 \\
\hline 4 & 0.1324 & 0.20 & $6 "$ & 0.25 & 0.75 & 7.8 & 35 & 0.1310 & -0.0014 \\
\hline
\end{tabular}

Nr. 1 und 2 gaben schwammige Niederschläge; die Flüssigkeit von Nr. 4 gab bei der Prüfung mit Schwefelwasserstoff nach Beendigung der Elektrolyse keine Färbung; aber diese Reaktion scheint hier nicht sehr empfindlich zu sein. Die Bedingungen von Nr. 3 sind wohl die vorteilhaftesten.

\section{Bestimmungen in Oxalatlösungen.}

Einige qualitative Versuche unter den Versuchsbedingungen, die beim Kadmiumsulfat die wenigst unbrauchbaren Niederschläge gegeben hatten, wurden auch beim Chlorid angestellt. Die Ergebnisse waren gleichfalls nicht befriedigend, so dafs die Oxalatmethode ohne weitere Versuche aufgegeben wurde.

\section{Bestimmungen in Lösungen von Harnstoff und dergl.}

Einige qualitative Proben schienen zu zeigen, dafs Lösungen mit Harnstoff, Formaldehyd oder Acetaldehyd für die Bestimmung des Kadmiums aus seinem Chlorid sehr geeignet wären; doch ergaben weitere Versuche, dals dies in der Tat nicht der Fall ist. Unter diesen Bedingungen wird das Kadmium viel schneller aus einer Chloridlösung als aus der Sulfatlösung gefällt und der Niederschlag scheint während der ersten Periode der Elektrolyse sehr 
brauchbar zu sein. Das frei gemachte Chlor wirkt jedoch offenbar auf die vorhandenen organischen Substanzen ein unter Bildung von Verbindungen, die für die Ausfällung schädlich sind. Bei grofser Sorgfalt lassen sich allerdings zufriedenstellende Ergebnisse erzielen, wie man aus der folgenden Tabelle sieht.

Reihe A: Harnstoff.

\begin{tabular}{|c|c|c|c|c|c|c|c|c|c|c|}
\hline 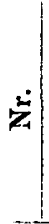 & 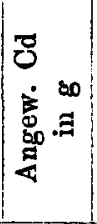 & 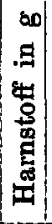 & 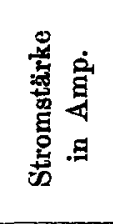 & 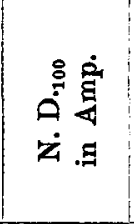 & 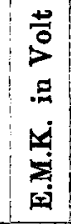 & 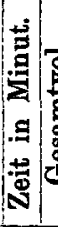 & : & 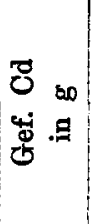 & 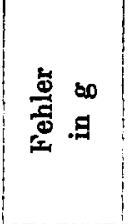 & Bemerkungen \\
\hline 1 & 0.1324 & & 1.0 & 3.0 & 12 & 206 & 60 & 0.1312 & -0.0012 & $\begin{array}{l}\text { schwammig, nicht alles } \\
\text { gefällt }\end{array}$ \\
\hline 2 & 0.1324 & 2 & 1.0 & 3.0 & 12 & 306 & 600 & 0.1336 & +0.0012 & $\begin{array}{l}\text { schwammig, nicht alles } \\
\text { gefällt }\end{array}$ \\
\hline 3 & 0.1324 & 3 & 1.0 & 3.0 & 7.8 & 15 & 550 & 0.1342 & +0.0018 & $\begin{array}{l}\text { sehr schwammig, alles } \\
\text { gefällt }\end{array}$ \\
\hline 4 & 0.1324 & & 0.25 & 0.75 & 7.8 & 25 & 60 & 0.1324 & \pm 0.0000 & alles gefällt \\
\hline 5 & 0.1324 & & 0.5 & 1.5 & 12 & 206 & 60 & 0.1333 & +0.0009 & $\begin{array}{l}\text { etwas schwammig, alles } \\
\text { gefällt }\end{array}$ \\
\hline 6 & 0.1324 & & 1.0 & $\mathbf{3 . 0}$ & 12 & 206 & 60 & 0.1370 & +0.0046 & sehr schwammig \\
\hline 7 & 0.1324 & 1.5 & $0.25-0.5$ & $0.75-1.5$ & 7.8 & 30,6 & & 0.1328 & +0.0004 & gut \\
\hline 80 & 0.1324 & 1.5 & $0.25-0.5$ & $0.75-1.5$ & 7.8 & 306 & 600 & 0.1329 & +0.0005 & ziemlich gut \\
\hline
\end{tabular}

Es sind demach zwischen 1.5-2 g Harnstoff anzuwenden und die Stromspannung soll 8 Volt nicht übersteigen, anstatt der beim Sulfat zulässigen 12 Volt. Die Reaktion mit Schwefelwasserstoff scheint in dieser Lösung nicht sehr empfindlich zu sein, so dafs man bei jeder Bestimmung wenigstens 30 Minuten elektrolysieren mufs. - Einige Autoren empfehlen zur Prüfung des Endpunktes der Fällung, das Niveau der Flüssigkeit um die Kathode zu erhöhen; dies ist hier aber nicht sehr zweckmälsig, da das auf der frischen Kathodenfläche abgeschiedene Metall am Ende der Elektrolyse fast nicht $\mathrm{zu}$ bemerken ist.

Eine Lösung mit Formaldehyd gab die folgenden Resultate:

\section{(S. Tabelle, S. 18.)}

Es folgt hieraus, dafs es zweckmälsiger ist, eine etwas geringere Menge Formaldehyd zu verwenden als beim Sulfat. Die Stromspannung soll 8 Volt nicht übersteigen; zweckmälsig nimmt man ein etwas grölseres Volumen $(60 \mathrm{ccm})$.

z. anorg. Chem. Bd. 47. 
Reihe C: Formaldehyd (Formalin).

\begin{tabular}{|c|c|c|c|c|c|c|c|c|c|c|}
\hline$\dot{z}$ & 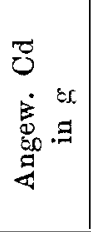 & 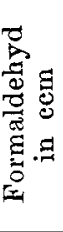 & 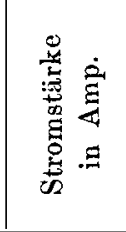 & 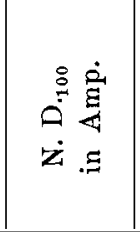 & 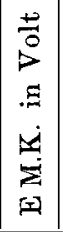 & 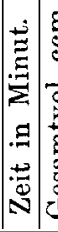 & 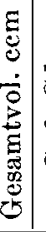 & $\mid \begin{array}{cc} & \\
0 & \\
0 & 50 \\
\dot{0} & .7 \\
b & \end{array}$ & 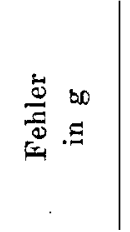 & Bemerkungen \\
\hline 1 & 0.1324 & 3.0 & $0.25-1.5$ & $0.75-4.5$ & 7.8 & $|20| 4$ & $45 \mid 0$ & 0.1333 & +0.0009 & \multirow{4}{*}{$\begin{array}{l}\text { ziemlich gut } \\
\text { etwas schwammig } \\
\text { dicht } \\
\text { dicht }\end{array}$} \\
\hline 2 & 0.1324 & 2.0 & $0.50-2.0$ & $1.50-6.0$ & 11,8 & \begin{tabular}{|l|l}
15 & 4
\end{tabular} & $45 \mid 0$ & $0.1330 \mid$ & +0.0006 & \\
\hline 3 & 0.1324 & 1.5 & 0.75 & 2.25 & 7.8 & $30 \mid 6$ & 600 & 0.1324 & \pm 0.0000 & \\
\hline 4 & 0.1324 & 1.5 & 1.0 & 3.0 & 7.8 & \begin{tabular}{|l|l|}
35 & 6
\end{tabular} & $60 \mid 0$ & 0.1325 & +0.0001 & \\
\hline
\end{tabular}

Noch notwendiger ist vorsichtiges Arbeiten beim Acetaldehyd, wenn zufriedenstellende Ergebnisse erhalten werden sollen.

Reihe C: Acetaldehyd (95\%).

\begin{tabular}{|c|c|c|c|c|c|c|c|c|c|}
\hline z & 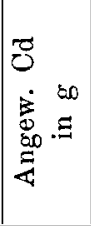 & 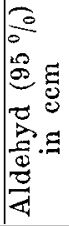 & 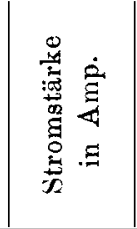 & 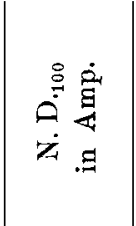 & 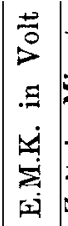 & 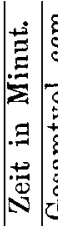 & 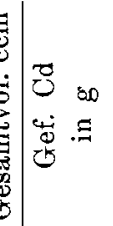 & 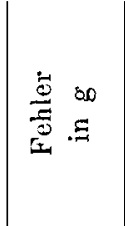 & Bemerkungen \\
\hline 1 & 0.1324 & 2.0 & $0.2-0.7$ & $0.6-2.1$ & 7.8 & & \begin{tabular}{l|l|l}
0 & 0.1311
\end{tabular} & -0.0013 & $\begin{array}{l}\text { nicht alles gefällt; } \\
\text { etwas schwammig }\end{array}$ \\
\hline 2 & 0.1324 & 1.5 & $\begin{array}{ll}0.2 & 0.75\end{array}$ & $0.6-2.25$ & 7.8 & 306 & 0.1346 & +0.0022 & schwammig \\
\hline 3 & 0.1324 & 0.5 & $0.2-1.0$ & $0.6-3.0$ & 7.8 & 656 & 300.1307 & -0.0017 & $\begin{array}{l}\text { schwammig, nicht } \\
\text { alles gefällt }\end{array}$ \\
\hline 4 & 0.1324 & 1.0 & $|0.1-0.75|$ & $|0.3-2.25|$ & 8.0 & $|35| 6$ & \begin{tabular}{l|l|l|}
$\mathbf{0}$ & 0.1328
\end{tabular} & $|-0.0001|$ & guter Niederschlag \\
\hline
\end{tabular}

\section{vIII. Bestimmungen in Lösungen von Formiaten und Tartraten.}

Wie Kadmiumsulfat, so gab auch Kadmiumchlorid ungünstige Resultate bei der Elektrolyse von Lösungen, die neben Kaliumformiat Ameisensäure enthielten. Auch wenn Formiat fehlte, waren in ameisensauren Lösungen die Ergebnisse nicht zufriedenstellend. - Eine Lösung von $0.1324 \mathrm{~g}$ Kadmium in Form von Chlorid wurde mit $1.5 \mathrm{ccm}$ Ameisensäure versetzt, das Ganze auf $50 \mathrm{ccm}$ verdünnt, und die Elektrolyse bei 7.5 oder 11.8 Volt ausgeführt. In beiden Fällen war der Niederschlag schwammig und haftete nicht, während etwas 
Kadmium in Lösung blieb, auch als der Strom fast zwei Stunden hindurchging.

Weinsäurehaltige Lösungen verhielten sich ähnlich. In Gegenwart von $3 \mathrm{~g}$ Weinsäure - bei Spannungen von 8 und 12 Volt schälte sich das gefällte Metall während der Drehung von der Kathode ab; der Niederschlag war schwammig, in keinem Stadium der Operation schien die Fällung vollständig zu sein.

The Kent Chemical Laboratory of Yale University, New Haven, U. S. A.

Bei der Redaktion eingegangen am 30. Juli 1905. 